\title{
Ready or Not (To Apply)? Autoregulatory Dysfunction Indices and Lesion Progression in Traumatic Brain Injury
}

\author{
Susanne Muehlschlegel ${ }^{*}$ (i)
}

C 2019 Springer Science+Business Media, LLC, part of Springer Nature and Neurocritical Care Society

When it comes to intracranial pressure (ICP) and cerebral perfusion pressure (CPP) targets in traumatic brain injury (TBI), the notion that "one size does not fit all" has been displayed by several important studies. For example, investigators from the Cambridge brain physics laboratory have first reported in 2002 [1] and recently validated in a large CENTER-TBI cohort in 2019 [2] that applying patient-individualized CPP and ICP targets using the relationship between ICP and the cerebrovascular pressure reactivity index (PRx), a moving correlation coefficient recorded over several minute periods between averaged values of MAP and ICP, is independently associated with better outcome than using guideline-recommended ICP thresholds. Also, the "BEST-TRIP trial," a large NIH-funded Phase-3 trial in severe TBI, showed that maintaining a non-individualized ICP at $20 \mathrm{mmHg}$ or less for all patients was not superior than imaging and clinical examination [3]. Nonetheless, real-time or just even retrospective PRx monitoring remains limited to very few neuro-ICUs around the world, due to practical reasons: the need for high-resolution monitoring, which requires hardware and software additions (read: costs for

*Correspondence: susanne.muehlschlegel@umassmed.edu Departments of Neurology, Anesthesiology and Surgery, University of Massachusetts Medical School, 55 Lake Ave North, S-5, Worcester, MA, USA

Invited Commentary to manuscript NECA-D-19-00349R3: "Relationship between measures of cerebrovascular reactivity and intracranial lesion progression in acute TBI patients: a exploratory analysis" by Mathieu et al. 2019. https://doi.org/10.1007/s12028-019-00885-3.

This comment refers to the article available at https://doi.org/10.1007/ s12028-019-00885-3. licensing, training and acquisition), technical expertise in software and hardware use, and time.

PRx and the notion of impaired autoregulation in TBI may be applied in other contexts. In this edition of Neurocritical Care, Mathieu et al. [4] publish their study testing their hypothesis that there may be a relationship between impaired autoregulation and traumatic lesion expansion in TBI. Their hypothesis is anchored in the fact that pathophysiological mechanisms for the association between PRx and outcome remain unclear in TBI, and that some data in animals suggest "that the energy transferred at the time of injury affects cerebrovascular responses in both the hemorrhagic lesion core and in perilesional zones and can impair microvascular function even in the absence of overt structural vascular damage" [4]. The authors' hopes are high for establishing a relationship between autoregulation impairment and traumatic lesion expansion. They ultimately hope to identify "a risk factor for lesion expansion, establishing a proximate structural consequence of physiological derangements, informing the frequency and timing of follow up imaging, and stratifying patients at high risk of needing surgical intervention and more aggressive medical therapies." [4] The objective of the study was to explore the relationship between PRx, the pulse amplitude index (PAx), and the correlation coefficient between the pulse amplitude of ICP and CPP (RAC), and traumatic lesion progression in patients with acute TBI. Their goal was also to find the indices with the strongest association to determine the best candidates for a larger confirmatory study. The authors carried out a retrospective study of 50 TBI patients undergoing multimodal intracranial monitoring between 2006 and 2014, who had at least 2 head computed tomography $(\mathrm{CT})$ scans performed in the

\section{Springer}


acute phase of injury. Patients were managed according to the TBI guidelines in effect during the study period with an ICP goal $<20 \mathrm{mmHg}$ and $\mathrm{CPP}>60 \mathrm{mmHg}$. Because of a delay in the start of physiological recording data and repeat CT, only 23 patients were analyzed. "Traumatic lesions" included all types of hemorrhagic lesions (traumatic SAH, contusion, epidural hematoma, subdural hematoma) and perilesional edema. Patients with radiological progression versus stability of intracranial lesions were compared. Correlations between the volumetric measurements of traumatic lesions and PRx, Pax, and RAC were found. The authors found some linear associations between PRx impairment and progression of pericontusional edema, with strongest correlations found between \%time $\mathrm{PRx}>0.25 \quad(r=0.69)$ and \%time $\mathrm{PAx}>0.25(r=0.64)$. Both \%time PRx $>0.25$ and \%time spent PAx $>0.25$, but not \%time spent RAC $>-0.10$, were associated with pericontusional edema progression, even after adjustment for baseline characteristics, mean CPP, and non-physiological factors associated with edema progression. Similar correlations were found with RAC and pericontusional edema. No correlations were found with the progression of the hemorrhagic core, total contusion volume, and extra-axial hemorrhage volume. There was concern for multiple comparisons; hence, the authors adjusted their $p$ values using the Bonferroni method.

This study shows, for the first time, a signal to raise the idea that there may be a potential association cerebrovascular reactivity (representing global autoregulatory dysfunction) and traumatic pericontusional edema progression. Strengths of the study include a rigorous and validated method of determining PRx and the other indices by a very experienced group of brain physics researchers. The authors challenged the unexplored boundaries of connecting autoregulatory dysfunction to traumatic radiological lesion progression. For both, the authors should be applauded. Nonetheless, the study and its findings remain highly exploratory. First, the reduction of an already small sample size due to the timing of monitoring start points out the difficulties inherent with PRx monitoring. To obtain PRx, the patient needs to have an ICP monitor and an arterial line placed, and then must be hooked up to hardware and the ICM+software, followed by the requirement of several epochs of continuous monitoring to even obtain a PRx. At least in the hyperacute setting, where traumatic lesion progression is at its highest risk, timing of monitoring start will limit its application. Second, the authors made a very large number of comparisons. While they Bonferronicorrected all $p$ values, any studies using this large number of comparisons raise the notion of looking for a "needle in the haystack," rather than testing a specific hypothesis. For sure the findings warrant replication in a larger cohort, validation in an external cohort, and the careful examination of the associations with outcome.

While mathematical correlation analyses are easy to carry out once the data has been obtained, the biggest questions remain: Why should we care? How will it change what we do? And how can patient outcomes be improved? Reassuringly, a three-center prospective, feasibility study is underway to determine the feasibility of obtaining and applying individualized, physiological brain parameters such as CPP-Opt and PRx to patients in a pragmatic way (COGITATE-trial [ClincalTrials. gov NCT02982122]). Feasibility results may be available as early as 2020 . Until then, exploratory studies in neurocritical care such as this one are necessary to help us look beyond our known horizons. Authors, reviewers, and editors must be vigilant to ensure that such studies are very carefully interpreted, pointing out the "exploratory" aspect of such studies not only in the manuscript itself, but also in the title and abstract, as the authors of this study [4] have rightfully done.

\section{Funding}

Dr. Muehlschlegel is supported by Grants NIH/NICHD 5K23HD080971 from Eunice Kennedy Shriver National Institute of Child Health and Human

Development and the PACE-Award 2018-2020 from UMassMemorial Medical Center/UMass Memorial Medical Group.

\section{Conflicts of interest}

Dr. Muehlschlegel has no conflict of interest.

\section{Publisher's Note}

Springer Nature remains neutral with regard to jurisdictional claims in published maps and institutional affiliations.

Published online: 16 December 2019

References

1. Steiner LA, Czosnyka M, Piechnik SK, et al. Continuous monitoring of cerebrovascular pressure reactivity allows determination of optimal cerebral perfusion pressure in patients with traumatic brain injury. Crit Care Med. 2002;30:733-8.

2. Zeiler FA, Ercole A, Cabeleira M, et al. Patient-specific ICP epidemiologic thresholds in adult traumatic brain injury: a CENTER-TBI validation study. J Neurosurg Anesthesiol. 2019. https://doi.org/10.1097/ANA.0000000000 000616.

3. Chesnut RM, Temkin N, Carney N, et al. A trial of intracranial-pressure monitoring in traumatic brain injury. N Engl J Med. 2012;367:2471-81.

4. Mathieu F, Zeiler FA, Whitehouse DP, et al. Relationship between measures of cerebrovascular reactivity and intracranial lesion progression in acute TBI patients: an exploratory analysis. Neurocrit Care. 2019. https:// doi.org/10.1007/s12028-019-00885-3 